\title{
Fetal Cerebral Ventriculomegaly: Sonographic Diagnostic Workup
}

\author{
Vincenzo D'Addario, Cristina A Rossi \\ Fetal Medicine Unit \\ Department of Obstetrics and Gynecology \\ University Medical School, Bari, Italy \\ Corresponding address: Prof. Vincenzo D’Addario, Fetal Medicine Unit, Dept. of Obstetrics and Gynecology, Ospedale \\ Policlinico, Piazza G. Cesare, 70124 Bari, Italy, \\ E-mail:v.daddario@gynecology4.uniba.it
}

\begin{abstract}
Dilatation of the fetal cerebral ventricles (ventriculomegaly) is a generic sonographic sign, which is common to several pathological entities carrying different prognosis.

V entriculomegaly is easily recognized by ultrasound by measuring the atrial width. This simple measure allows the recognition of even mild forms of ventricular dilatation and is used as a screening method for ventriculomegaly. However, although the diagnosis of ventriculomegaly is easy, the prenatal identification of the cause of ventricular dilatation is a more difficult task. The recognition of associated brain anomalies is a crucial point. The research of the cause of ventriculomegaly is clinically useful, since the prognosis mainly depends on the etiology and on the presence of associated abnormalities.

In this article the role of prenatal sonography in recognizing the cause of the ventriculomegaly is reviewed.
\end{abstract}

Keywords: Aqueductal stenosis, Chiari II malformation, corpus callosum agenesis, Dandy-Walker complex, hydrocephalus, prenatal diagnosis, ultrasound, ventriculomegaly

Fetal cerebral ventriculomegaly is a sonographic sign common to different pathological entities. The incidence of congenital ventriculomegaly ranges from 0.3 to 1.5 in 1000 births in different series. ${ }^{1}$ The term "hydrocephalus" is frequently used as synonymous; however the term "ventriculomegaly" should be preferred since it identifies the abnormal sonographic finding, independently from the etiology.

The ventricular dilatation may be a consequence of the increased pressure of the cerebrospinal fluid (true hydrocephalus) due to obstruction of cerebrospinal flow (obstructive hydrocephalus) or, less frequently, to hypersecretion or defective filtration of cerebrospinal fluid. V entriculomegaly may also be the consequence of an altered development of the intracranial architecture without increase of the fluid pressure: a typical example of this condition is the agenesis of the corpus callosum, where the posterior expansion of the lateral ventricles derives from the distorted array of the white matter tracts in the occi pital lobes. $^{2}$

The main causes of ventricular dilatation in the fetus are aqueductal stenosis (33-43\% of the cases), Chiari II malformation (25-30\%), Dandy-Walker complex (7-10\%), dysgenesis of the corpus callosum (20-30\%). Other brain anomalies less frequently associated with ventriculomegaly are: occupying space lesions (tumors and cysts), haemorrhage, neuronal proliferation and differentiation disorders. Finally isolated mild ventriculomegaly may be found, without any clinical sequelae.

A queductal stenosis is the most common cause of fetal ventriculomegaly. It can be caused by infections (toxoplasmosis, cytomegalovirus), bleeding or other pathologies that cause gliosis and subsequent obliteration of the aqueduct. Sometimes the aqueduct can be congenitally atresic or few rudimentary ependymal-lined tubules can be seen in place of it (aqueductal forking); in these cases a multifactorial pattern of inheritance is probably the cause, but in $5 \%$ of all cases an X-linked transmission is present (recurrence risk $50 \%$ of males). $X$-linked hydrocephalus is due to mutations of a gene on $X q 28$ that encodes for $L 1$, a neural cell adhesion molecule. ${ }^{3}$

Chiari II malformation is a complex abnormality consisting in various combinations of brainstem and cerebellar malformations, associated with ventriculomegaly and spinal defect. In Chiari II malformation the ventricular dilatation is the final result of a cascade of events on the developing brain, starting from the primitive spinal defect: the leakage of the cerebrospinal fluid through the defect causes a decompression and collapse of the ventricular cavities; failure of the rombencephalic cavity to distend causes the development of a small posterior fossa that is inadequate to house the developing cerebellum which herniates caudal ly into the foramen magnum, causing obstruction of the outlets of the fourth ventricle, 
stretching and narrowing of the aqueduct, obliteration of the cisterna magna, obstruction at the level of dysplastic tentorium and consequently ventriculomegaly. ${ }^{4}$ D ue to its mechanism of development, ventriculomegaly is present only in $70-80 \%$ of fetuses affected by $\mathrm{C}$ hiari II malformation.

Dandy-Walker complex is a spectrum of posterior fossa abnormalities, the key feature of which is a variable severity of defect in the development of the cerebellar vermis (from complete agenesis to mild hypoplasia). ${ }^{5}$ In the most severe forms the fourth ventricle is extremely dilated and the membrane that forms its roof balloons, creating a large posterior fossa cyst. This cyst pushes the tentorium and the venous sinus running through it, causing obstruction of the cerebrospinal fluid and ventriculomegaly.

A genesis of the corpus callosum consists in a complete or partial absence of the white fibres that cross the midl ine between the two hemispheres. The etiology is heterogeneous, but genetic factors are predominant. Over 90 genetic syndromes are known that may present partial or total agenesis of the corpus call osum. ${ }^{6}$ This anomaly is almost constantly associated with ventricular dilatation of varying severity, but without increased intraventricular pressure. The ventriculomegaly is the consequence of an altered development of the intracranial architecture and derives from the distorted array of the white matter tracts in the occipital lobes.

\section{SONOGRAPHIC DIAGNOSIS OF FETAL CEREBRAL VENTRICULOME GALY}

The fetal brain and particularly the lateral ventricles undergo extensive structural changes during gestation, thus offering a variety of sonographic findings. Although the description of the normal appearance of the lateral ventricles was already reported in the 80 's mainly based on the axial scans of the fetal head, ${ }^{7,8}$ the first systematic reports on the fetal brain morphology, including ventricular system, evaluated on sagittal and coronal scans during both early and late pregnancy were published in 1991 by Timor-Tritsch's group. ${ }^{9,10}$ They proposed to use the transvaginal approach to evaluate the fetal brain through the anterior fontanella or any open suture, thus obtaining a series of sagittal and coronal planes comparable with those normally used by the neonatologist on the newborns.

Recently 3D US has also been applied to the evaluation of the fetal brain and ventricles. ${ }^{11,12}$ The main advantage of this technique is the possibility to store a sample volume of the brain and subsequently to reconstruct by means of the multiplanar imaging an infinite series of scans along the three orthogonal spatial planes (axial, sagittal and coronal) (Fig. 1).

The prenatal detection of fetal ventriculomegaly relies on the sonographic recognition and measurement of dilated lateral ventricles. Several measurement techniques have been suggested

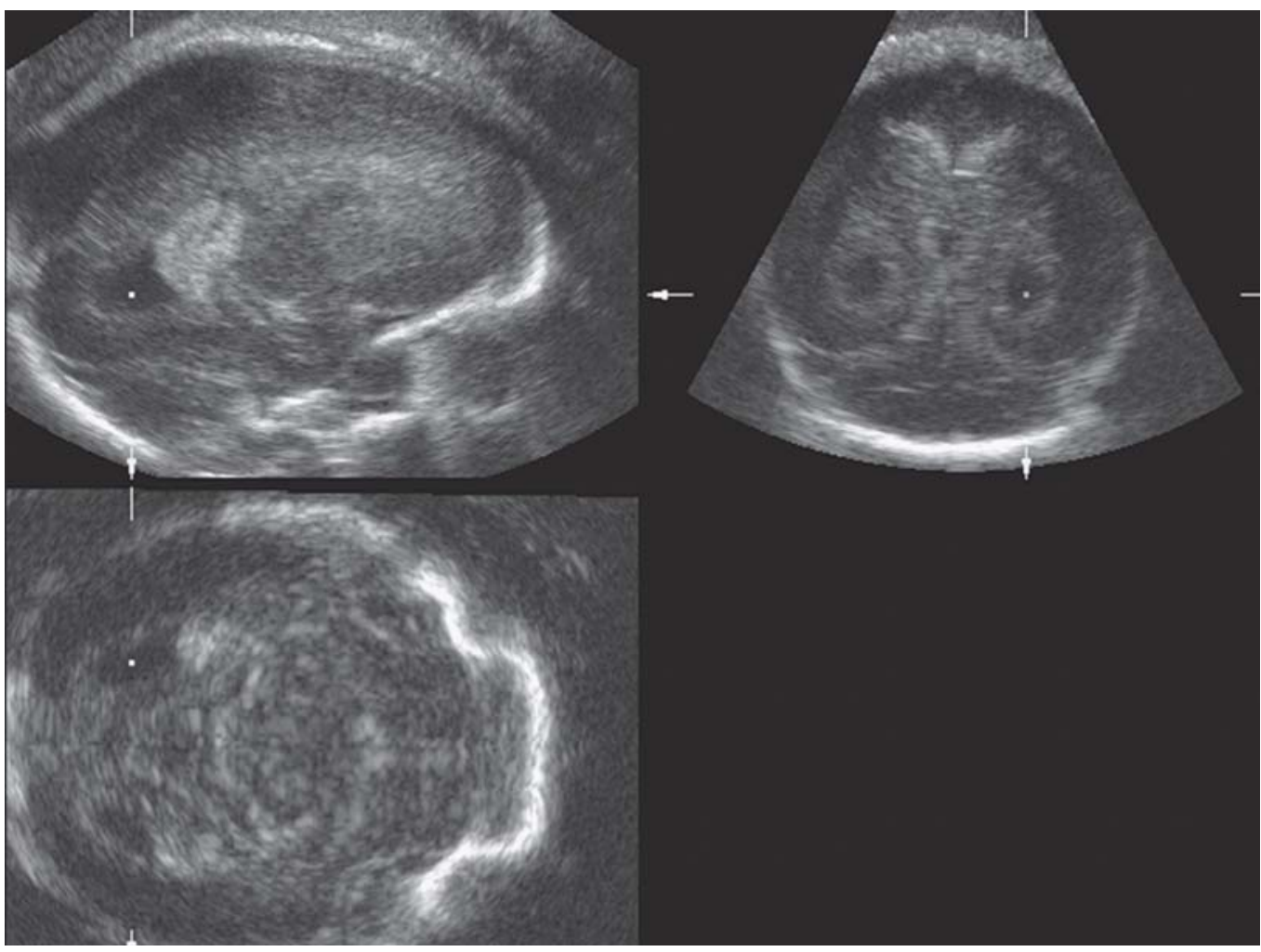

Fig. 1: Three-dimensional multiplanar view of a normal fetal brain, showing the normal lateral ventricle in the three spatial planes (sagittal, coronal and axial) intersecting at the level of the occipital horn 
starting from the lateral ventricle width/hemispheric width ratio, ${ }^{13}$ and moving to the atrial width measurement, ${ }^{14}$ till the multiple and fine measurements suggested by M onteagudo et al, ${ }^{15}$ obtainable by the transvaginal approach on the coronal and sagittal scans. A ctually the atrial width measured in the axial scan still is the best and easiest way to recognize ventricular dilatation and it is routinely used as a simple method to screen for ventriculomegaly. Measurements up to $10 \mathrm{~mm}$, independently from gestational age, are considered normal (Fig. 2A). A $n$ atrial width ranging from 10 to $15 \mathrm{~mm}$ is defined as borderline ventriculomegaly (Fig. 2B); measurements $>15 \mathrm{~mm}$ represent marked ventriculomegaly (Fig. 2C). A ctually, in cases of marked ventriculomegaly the diagnosis can be made directly without the need of measurements; these can be useful only to quantify and monitor the progression of the ventricular dilatation.

\section{RECOGNIZING THE CAUSE OF FETAL CEREBRAL VENTRICULOMEGALY}

When a cerebral ventriculomegaly is recognized it becomes important to rule out for associated abnormalities, chromosomopathies, infectious diseases and to recognize the cause of the ventricular dilatation, since from these factors mainly depends the prognosis and the parental counselling.

I solated ventriculomegaly due to aqueductal stenosis carries the less severe prognosis. In a series of 279 cases with isolated ventriculomegaly, 175 (63\%) survived; after shunting procedure 111 (63\%) were normal, the remainder having mild to severe neurological handicap. The survival rate in the 81 cases with additional anomalies was $23.5 \%$ (19 cases) and only 3 (15\%) of the survivors were normal, thus confirming the rel evant role played by the associated abnormalities ${ }^{16}$.

The prognosis of Chiari II malformation mainly depends from the severity of the associated spinal dysraphism that usually causes severe motor and sphyntherial defects. Despite these problems $75-85 \%$ of children after shunting procedure have normal intelligence.
The prognosis of hydrocephalic fetuses with D andy-W alker complex is poor but not uniformly fatal. The presence of other anomalies is associated with the worst prognosis. ${ }^{17}$

Counselling parents with fetuses affected by agenesis of the corpus callosum is a very difficult task since the prognosis is extremely variable. I solated agenesis of the corpus callosum may be either a completely asymptomatic event or can be characterized by subtle neurological deficits, revealed by specific tests. ${ }^{18}$ A genesis of the corpus callosum may be associated with other abnormalities or can be part of different syndromes: more than 90 conditions presenting agenesis of the corpus callosum have been reported. ${ }^{19}$ The outcome depends mainly on the presence of associated abnormalities.

$\checkmark$ entriculomegaly due to the presence of occupying space lesions, mainly depends from the nature of the lesion: it is usually good in arachnoid cysts which compress the adjacent brain, whereas it is usually poor in cases of tumors which infiltrate and distort brain anatomy.

As regards the severity of the ventricular dilatation as a prognostic factor, it seems to play a less important role than the cause of ventriculomegaly. The brain damage due to the increased pressure of the cerebrospinal fluid is a late event. Since the grey matter is compromised only in the late phase of the process, the measurement of the cerebral mantle thickness "per se" is not a reliable value to predict the severity of the neurological deficit.

A further important prognostic factor in fetuses with cerebral ventriculomegaly, apart from the cause of the ventricular dilatation, is the association with extra CNS abnormalities. A ssociated malformations have been found in 70 to $83 \%$ of the cases and $60 \%$ of the associated abnormalities are extracranial. ${ }^{20}$ The association with chromosomal defects is another important prognostic factor and the incidence of chromosomopathies is much higher in hydrocephalic fetuses with associated anomalies $(25-36 \%)$ than in fetuses with isolated ventriculomegaly (3$6 \%)^{21,22}$ Ruling out associated brain and extracranial anomalies is particularly important in cases of borderline ventriculomegaly: when the mild dilatation is truly isolated the neurological

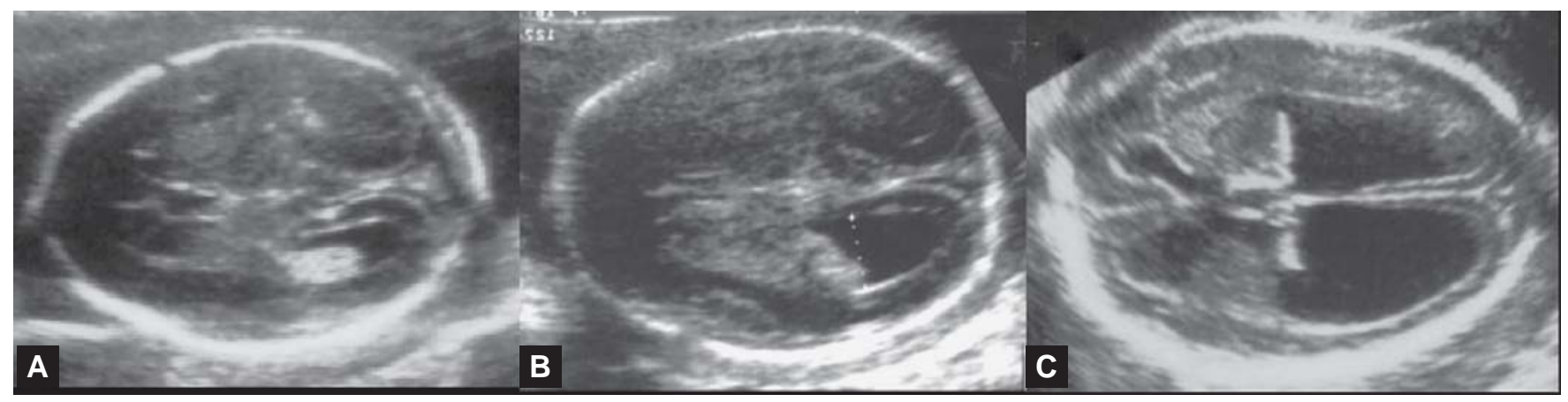

Fig. 2: Axial view of the brain at the level of the atrium of the lateral ventricle showing a normal case (A), a case of borderline ventriculomegaly (B) and a case of marked ventriculomegaly (C) 
handicap is good in $90-92 \%$ of the cases; ${ }^{23,24}$ the small percentage of bad outcome mainly affects the subgroup with atrial width between 12 and $15 \mathrm{~mm}^{25}$.

Considering the role that the cause of ventriculomegaly plays in influencing the prognosis, it appears evident how crucial is its prenatal recognition. A $n$ accurate evaluation of the shape of the dilated ventricles as well as the presence of associated intracranial abnormalities allows to obtain this goal in most cases.

The sonographic feature of aqueductal stenosis is that of tri-ventricular dilatation affecting the lateral and third ventricles with no other abnormality of the brain structures: for this reason it is also defined as isolated hydrocephalus (Fig. 3). The dilatation of the third ventricle is sometimes of little degree and the dilated lateral ventricles appear as the prominent feature. In cases of huge dilatation of the lateral ventricles, fenestration of the septum pellucid may occur (Fig. 4). Prenatal sonography

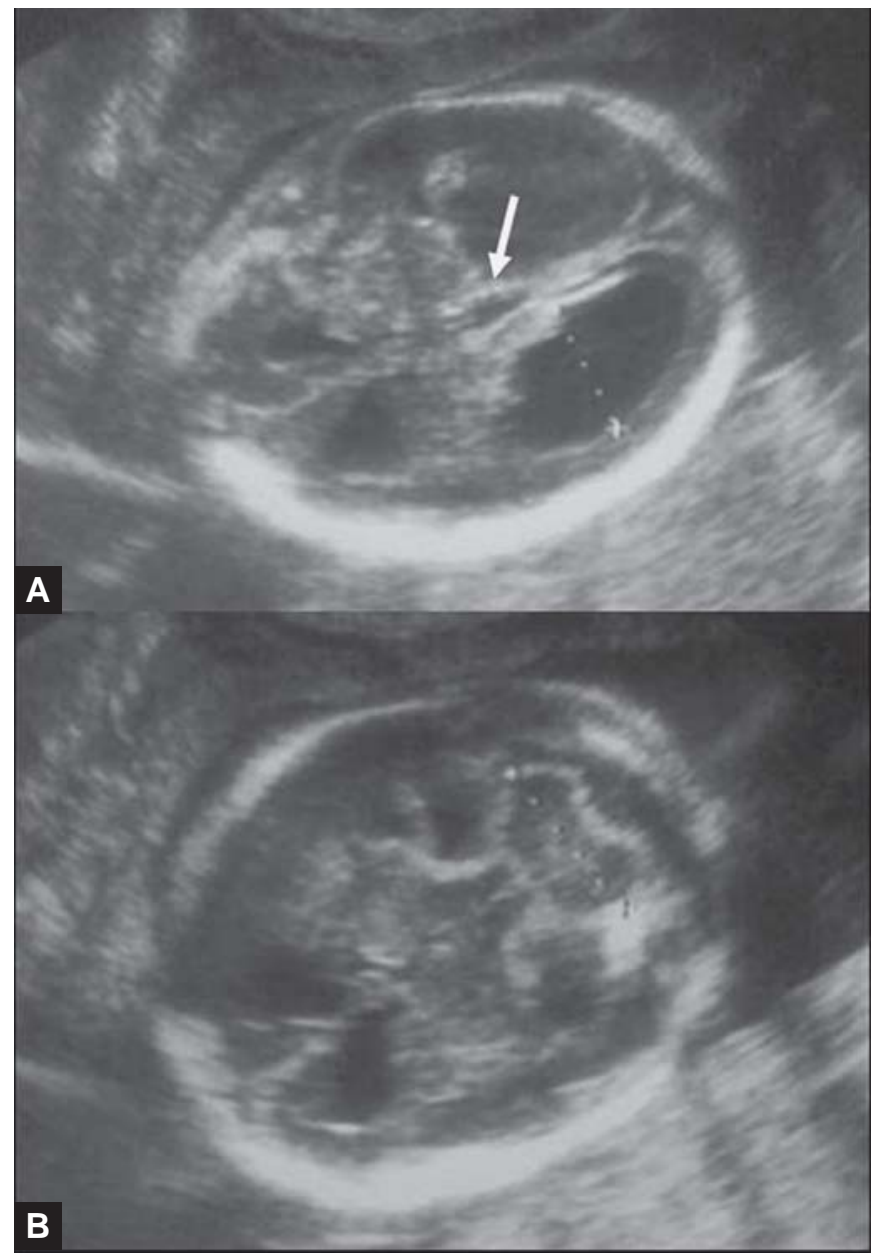

Figs $\mathbf{3 A}$ and B: Ventriculomegaly in a case of aqueductal stenosis. A marked dilatation of the lateral ventricles and a mild dilatation of the third ventricle (arrow) are visible. The remaining brain anatomy is normal does not permit to recognize the stenotic aqueduct, but the absence of any other abnormality in the brain authorizes to suspect this entity as the cause of the ventriculomegaly. Sonographic demonstration of abducted thumbs should prompt the diagnosis of $X$-linked hydrocephal us spectrum. ${ }^{26,27}$

In the Chiari II malformation, ventriculomegaly is the consequence of an al tered flow of the cerebrospinal fluid at the level of a small and funnelling posterior fossa associated with a spinal defect. The ultrasonographic appearance of the ventriculomegaly is not specific, but the axial scan on the posterior fossa shows that it is small, with cisterna magna being effaced and the cerebellum assuming the typical banana shape, secondary to its herniation in the foramen magnum ${ }^{28}$ (Fig. 5). In order to evaluate the shape and quantify the size of the posterior fossa, our group suggested to measure the angle between the clivus and the supraocciput obtained in a sagittal scan of the skull (Fig. 6); this angle has a value of $79.3^{\circ}+6^{\circ}$ independently from gestational age and fetuses affected by Chiari II malformation show values bel ow $5^{\circ}$ centile $\left(<72^{\circ}\right) .^{29}$

$V$ entriculomegaly is a common finding in fetuses affected by Dandy-W alker complex. In a series of 99 cases (50 DandyW alker and 49 D andy-W alker variant) Ecker et al. reported an association with ventriculomegaly in $32 \%$ and $27 \%$ respectively. ${ }^{17}$ Once again the sonographic appearance of the ventricular dilatation "per se" is not specific, but the presence of a "cyst" of variable size in the posterior fossa is highly characteristic: it is secondary to the complete or partial agenesis of the cerebellar vermis and to the cystic dilatation of the fourth ventricle (Fig. 7). ${ }^{17,30}$

The proportion of fetuses with agenesis of the corpus callosum that have also ventriculomegaly is uncertain. When present, the ventricular dilatation is usually mild to moderate, al though severe forms of ventriculomegaly may also develop. The diagnosis is usually late since the development of the corpus callosum is a late event that is complete only by midgestation. B ennet et al. affirm that the diagnosis is not possible before 22 weeks, ${ }^{31}$ but available experience suggests that agenesis of the corpus callosum can be suspected at 18-20 weeks. The ventriculomegaly is the consequence of an altered development of the intracranial architecture and derives from the distorted array of the white matter tracts in the occipital lobes: for this reason it is usual ly moderate and limited to the posterior horns, so that the lateral ventricles assume the typical "tear-drop" appearance. The corpus callosum cannot be demonstrated by the axial scans. B ut indirect signs useful for the diagnosis are; ${ }^{32}$

- the enlargement of the interhemispheric fissure where three parallel lines can be recognized (the fal $x$ and the medial borders of the separated hemispheres): this sign is due to the altered architecture of the sulci and gyri of the medial aspect of the hemispheres. 


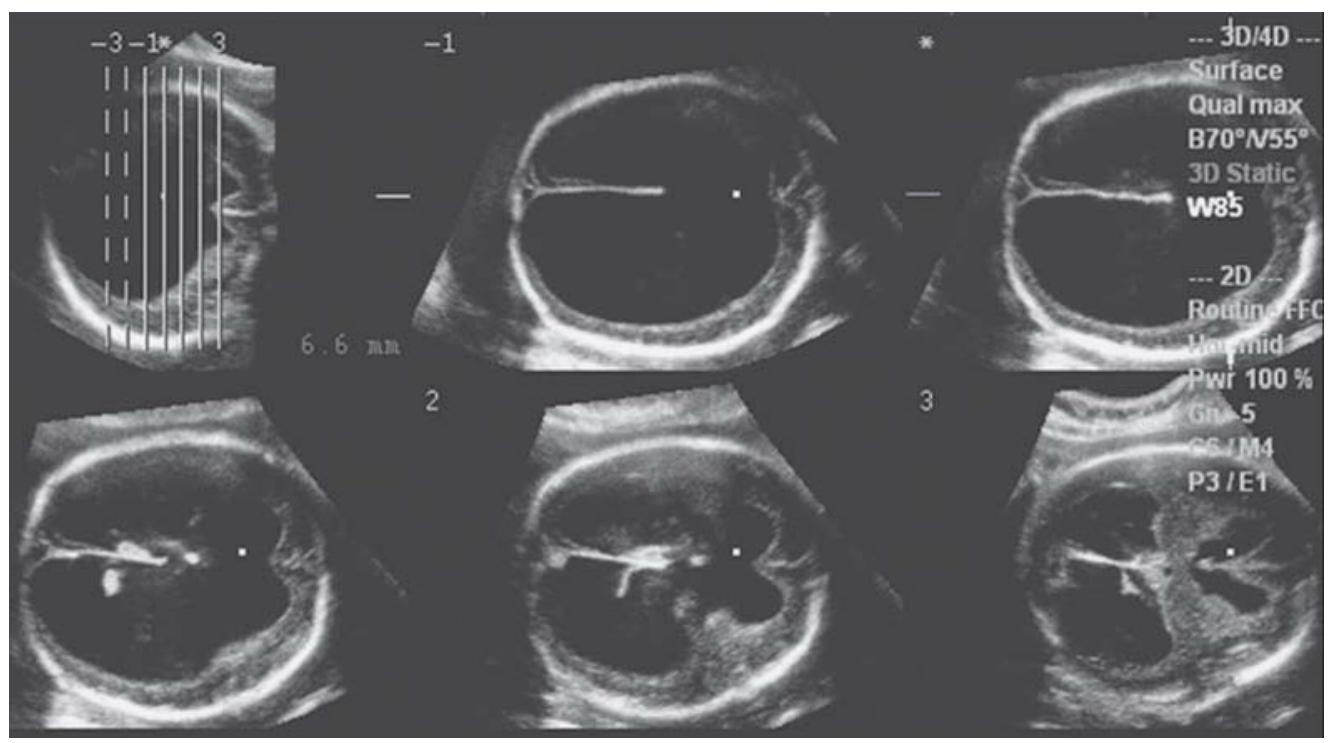

Fig. 4: 3D tomographic ultrasound imaging in the axial plane in a case of huge ventriculomegaly, showing the fenestration of the septum pellucidum (dots)

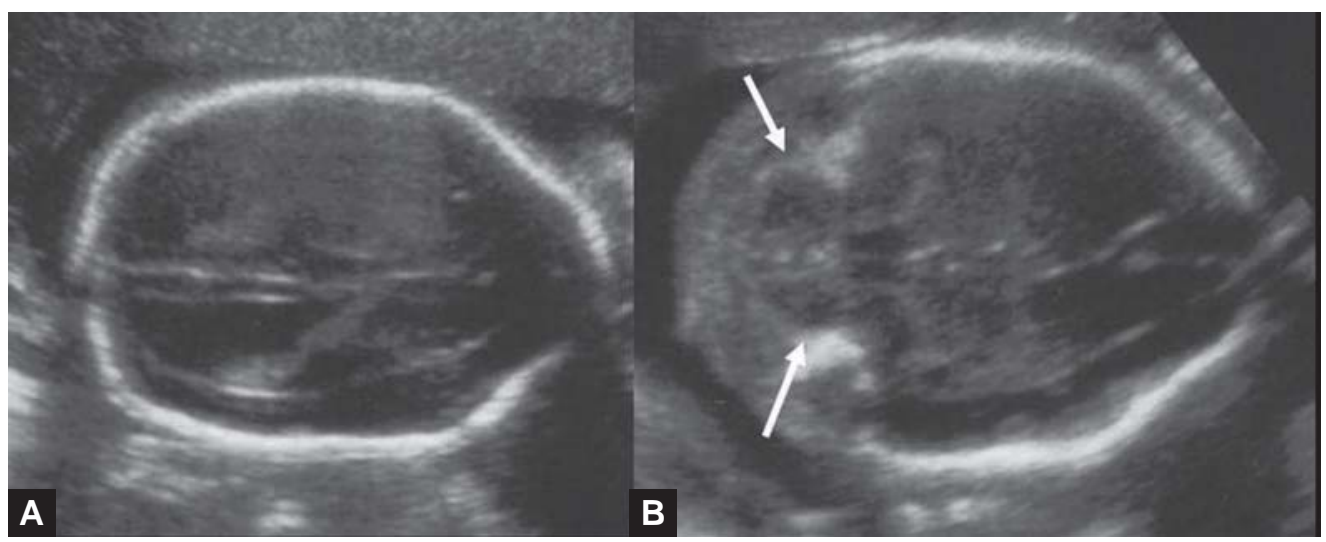

Figs 5A and B: Axial views of the fetal brain in a case of ventriculomegaly due to Chiari II malformation. (A) The ventriculomegaly and the typical "lemon" shaped skull are visible (B) The posterior fossa is small with effaced cisterna magna and with small and "banana" shaped cerebellum

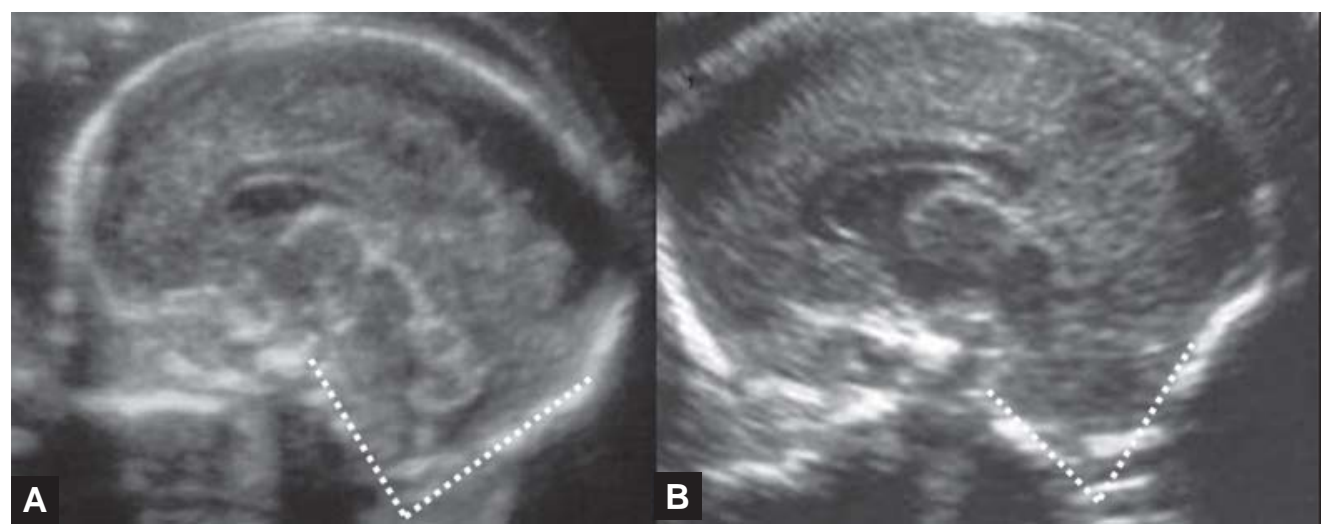

Figs 6A and B: Sagittal view of the brain in a normal case (A) and in a fetus affected by Chiari ll malformation (B). The funnelling appearance of the posterior fossa is pointed out by the smaller clivus-supraocciput angle as compared to the normal case 


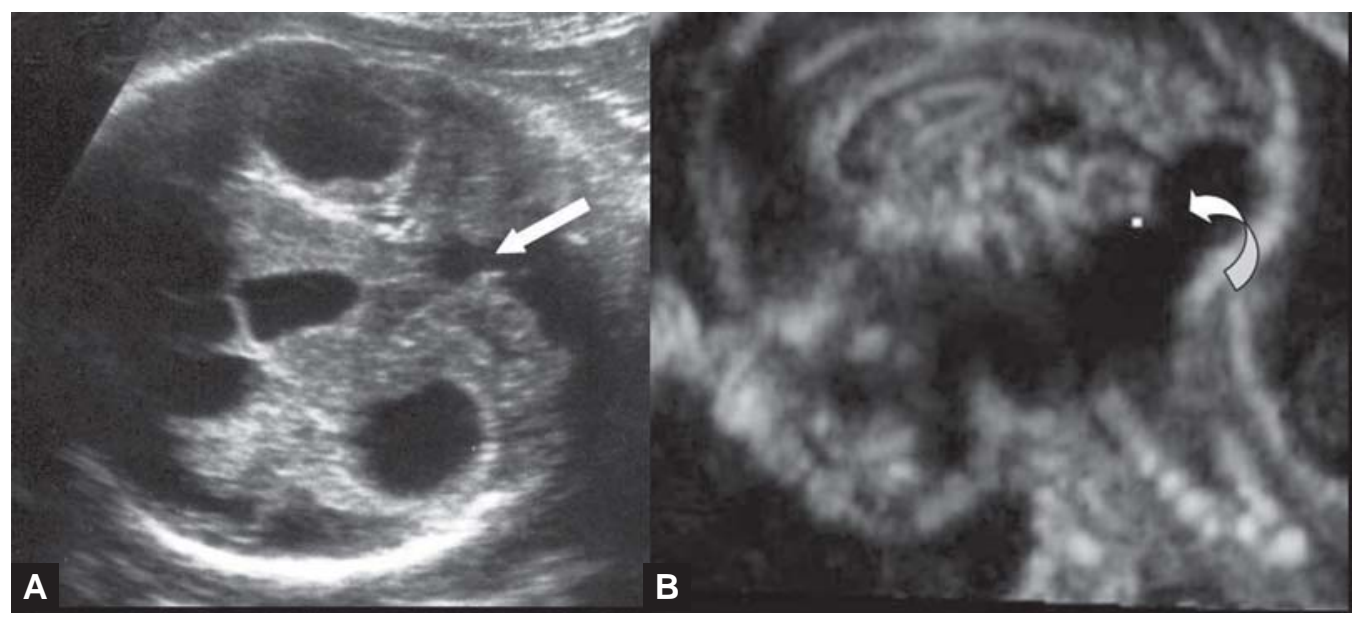

Figs 7A and B: (A) Axial scan of the brain in a fetus with ventriculomegaly due to Dandy-Walker malformation: the "cyst" in the posterior fossa and the absence of the cerebellar vermis are visible (arrow). (B) Sagittal view of the brain in a fetus affected by Dandy-walker malformation. The upward rotation of the hypoplastic vermis and the cystic dilatation of the fourth ventricle are clearly visible (curved arrow)

- the lateral displacement and enlargement of the bodies of the lateral ventricles, due to the medial compression of the white fibres failing to cross the midline.

- the upward displacement of the third ventricle (present only in $50 \%$ of the cases), sometimes communicating with an interhemispheric cyst, as a consequence of the absence of the corpus callosum (Fig. 8).

The definitive diagnosis relies on the demonstration of the absence of the complex formed by the corpus callosum and the cavum septi pellucidi. This sign can be achieved by the sagittal and coronal sections. The midsagittal section will demonstrate the compl ete or partial absence of the corpus callosum (Fig. 9). In cases of partial agenesis the missing part is the caudal one. In complete agenesis the gyri and sulci of the medial hemispheric surface show an atypical radiate appearance, due to the absence of the gyrus cinguli. W ith the use of color-doppler the absence of the pericallosal artery can be noticed. The coronal section at the level of the frontal horns will show their typical "bull-shape" appearance due to the medial compression by the white fibres that fail to cross the hemispheres (bundles of Probst) (Fig. 10).

Considering the sonographic findings described so far that characterize the different causes of ventricul omegaly, it appears evident that the differential diagnosis mainly relies on the evaluation of the posterior fossa and the corpus call losum. B oth structures can be evaluated in the midsagittal scan of the fetal brain. By combining the sonographic findings at the level of the posterior fossa and the corpus callosum it is possible to identify the main causes of fetal ventriculomegaly described so far.

When the posterior fossa is normal and the corpus callosum is present, the third ventricle is dilated and no other brain anomaly can be demonstrated, the ventriculomegaly is isolated and the most probable cause is aqueductal stenosis (Fig. 11B).

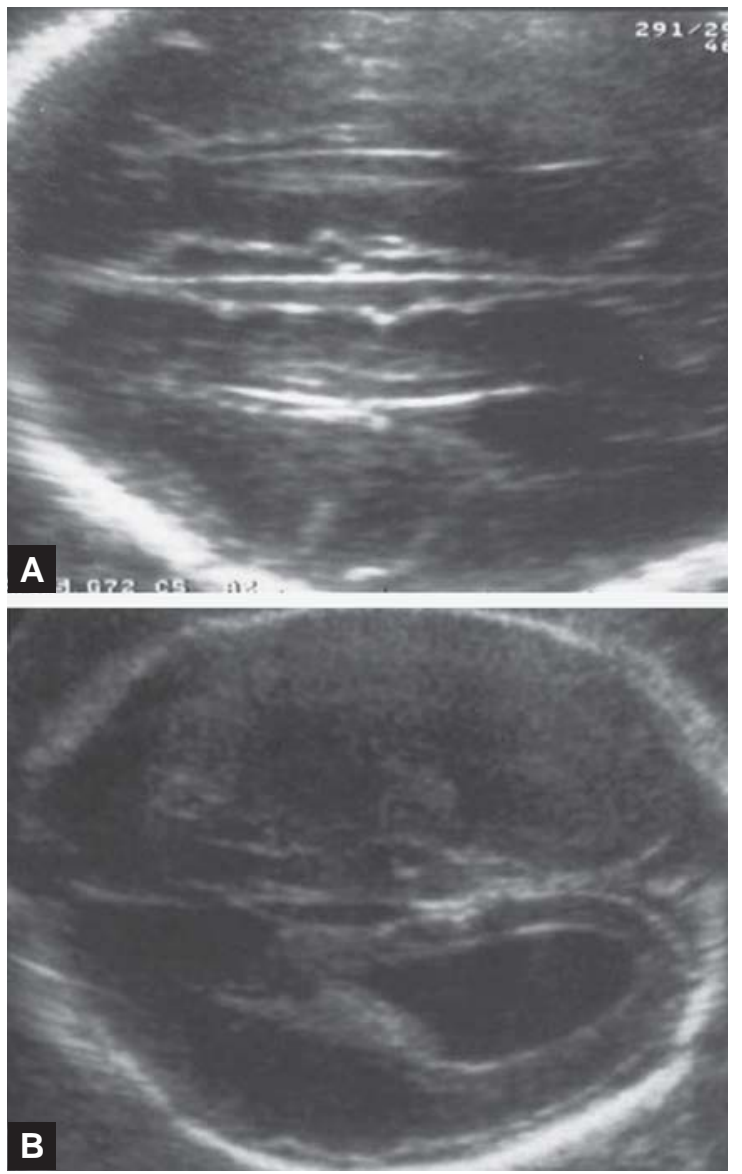

Figs $\mathbf{8 A}$ and $\mathbf{B}$ : Indirect signs of agenesis of the corpus callosum in the axial view: (A) enlargement of the interhemispheric fissure whit three parallel lines referring to the fal $x$ and the medial borders of the separated hemispheres. (B) Lateral displacement and enlargement of the bodies of the lateral ventricles 


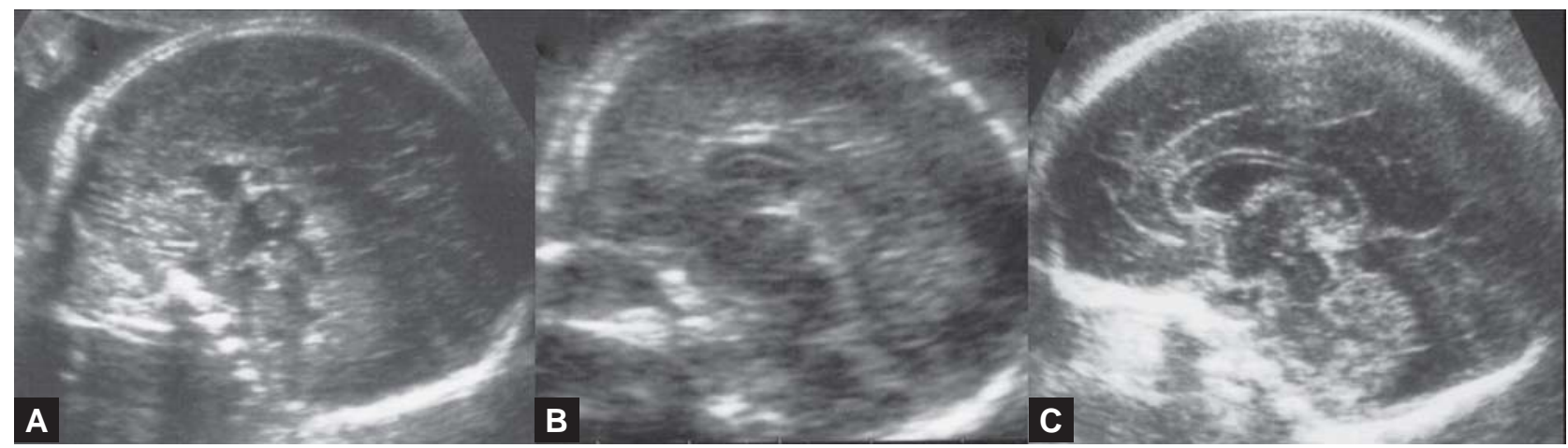

Figs 9A to C: Midsagittal view of the brain: (A) Complete agenesis of the corpus callosum (B) Partial agenesis of the corpus callosum (C) Normal corpus callosum

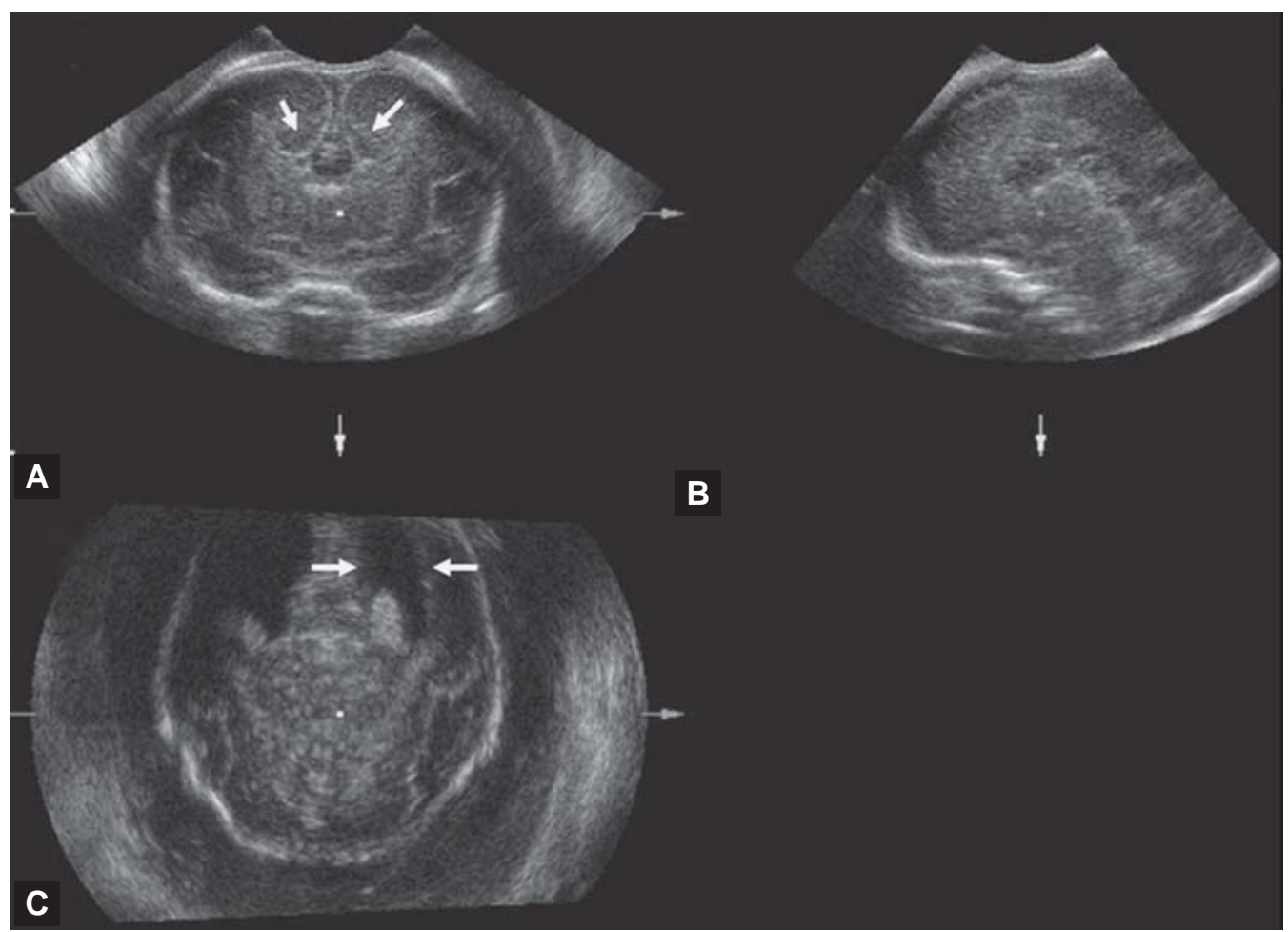

Figs $10 \mathrm{~A}$ to $\mathrm{C}$ : 3D multiplanar view of the brain in a fetus affected by agenesis of the corpus callosum: the coronal view (A) shows the typical "bull shape" appearance of the frontal horns (arrows); the sagittal view (B) shows the absence of the corpus callosum; the mild dilatation of the occipital horns (arrow) can be seen in the axial view (C)

When the posterior fossa is small with a clivus-supraocciput angle $<72^{\circ}$ and the cisterna magna is effaced, Chiari II malformation should be suspected as the cause of the ventricular dilatation (Fig. 11C) and a spinal defect should be carefully searched for.

When the posterior fossa shows a complete or partial agenesis of the cerebellar vermis with a cystic dilatation of the fourth ventricle, Dandy-Walker complex is the cause of the ventriculomegaly (Fig. 11D).
When the posterior fossa is normal and the corpus cal losum is completely or partially absent the ventriculomegaly is due to the failed devel opment of this structure (Fig. 11F).

A combinations of signs can of course be occasionally found; a typical association is that of agenesis of the corpus callosum with Dandy-Walker complex: both sonographic features can be achieved in the same sagittal scan (Fig. 11E).

3D facility and particularly the multiplanar view mode is extremely useful in "reconstructing" the midsagittal view once the volume from the fetal bain has been extracted. 


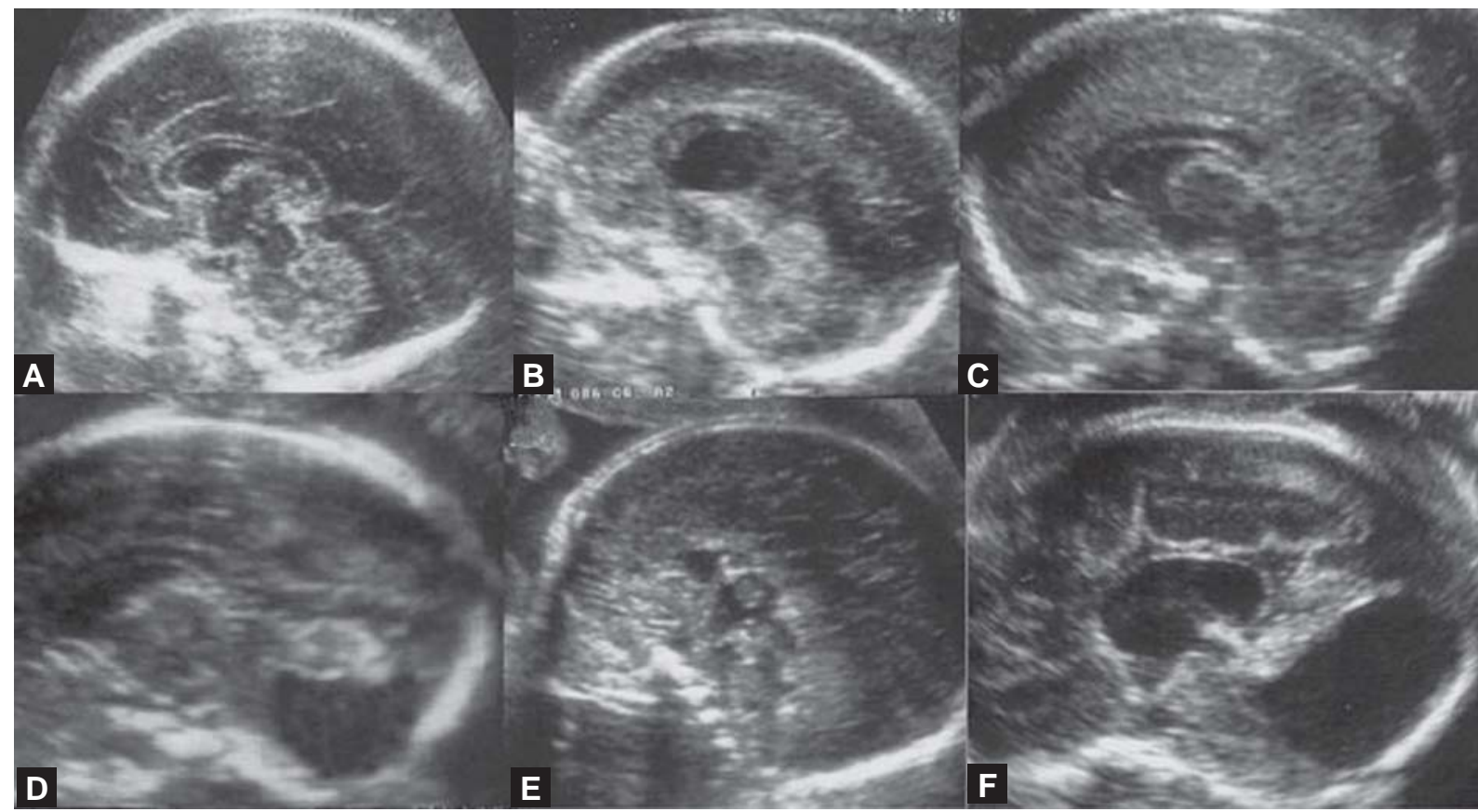

Figs 11A to F: Midsagittal view of the brain in different causes of ventriculomegaly: (A) normal brain: the normal corpus callosum and cerebellar vermis with fourth ventricle can be recognized. (B) Aqueductal stenosis: the posterior fossa is normal; the third ventricle is dilated; the corpus callosum is present. (C) Chiari II malformation : the posterior fossa is small with a clivus-supraocciput angle $<72^{\circ}$ and the cisterna magna is effaced. (D) Dandy-Walker complex : the posterior fossa shows a partial agenesis of the cerebellar vermis with a cystic dilatation of the fourth ventricle. (E) Agenesis of the corpus callosum: the posterior fossa is norma but corpus callosum is absent. (F) Combined agenesis of the corpus callosum and Dandy-Walker complex: the corpus callosum is absent, the third and the fourth ventricle are dilated

The accuracy of midsagittal scan in the diagnosis of the cause of ventriculomegaly is higher in cases with abnormal posterior fossa, lower in cases of isolated ventriculomegaly, due to the fact that the visualization of the corpus callosum is sometimes doubtful. I n a series of 58 cases of ventriculomegaly examined by our group, the midsagittal view of the fetal brain allowed to recognize accurately the cause of the ventricular dilatation in 8: $93.1 . \%$ of the cases. The only uncorrected diagnoses referred to four cases of partial agenesis of the corpus callosum, thus confirming the difficulties in recognizing this structure. ${ }^{33}$ The use of 3D multiplanar view hel ps in by-passing these technical problems.

Even though the midsagittal view of the brain is a powerful tool in the differential diagnosis of fetuses with ventriculomegaly, the diagnostic work-up should include also a careful evaluation of the finest anatomical details of the brain in order to recognize even rare cases of ventricular dilatation. Huge occupying space lesions, such as tumors or cysts, are easily recognizable (Fig. 12), but also small and subtle anomalies, such as cavum veli interpositi cyst (Fig. 13) or subependymal cysts (Fig. 14) may cause mild ventricular dilatation. ${ }^{34}$ The inner walls of the lateral ventricles should al so be carefully eval uated in order to recognize signs of intra- or extra-ventricular

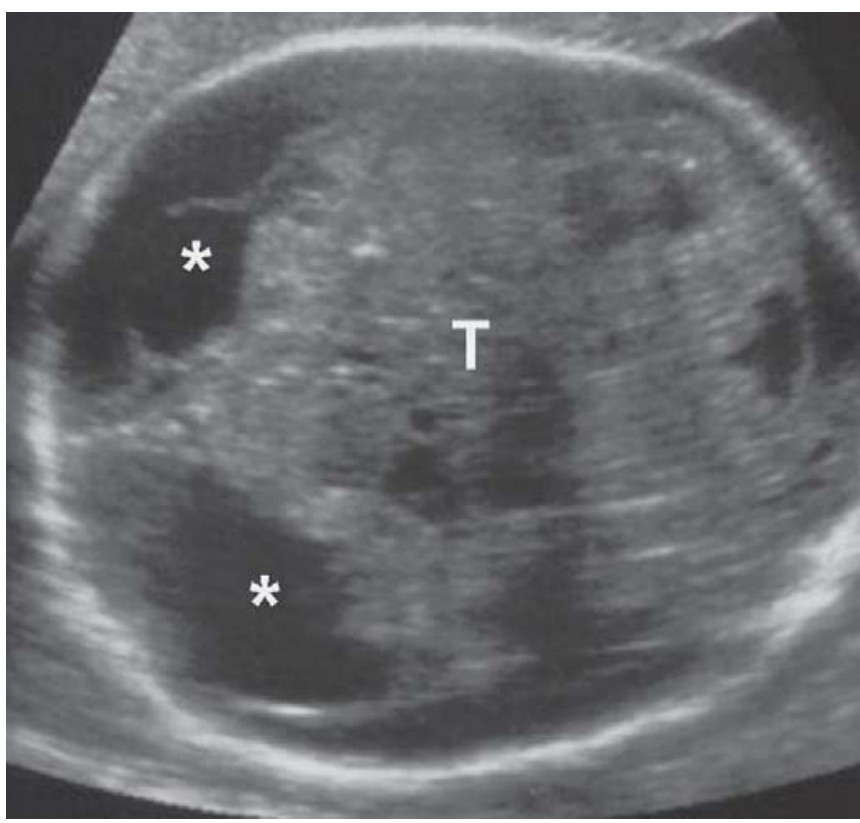

Fig. 12: Ventriculomegaly associated with brain teratoma. The tumor $(T)$ is easily recognizable as an echogenic mass distorting the brain anatomy and causing asymmetrical ventriculomegaly $\left({ }^{*}\right)$ hemorrhage (Fig 15 and 16). 


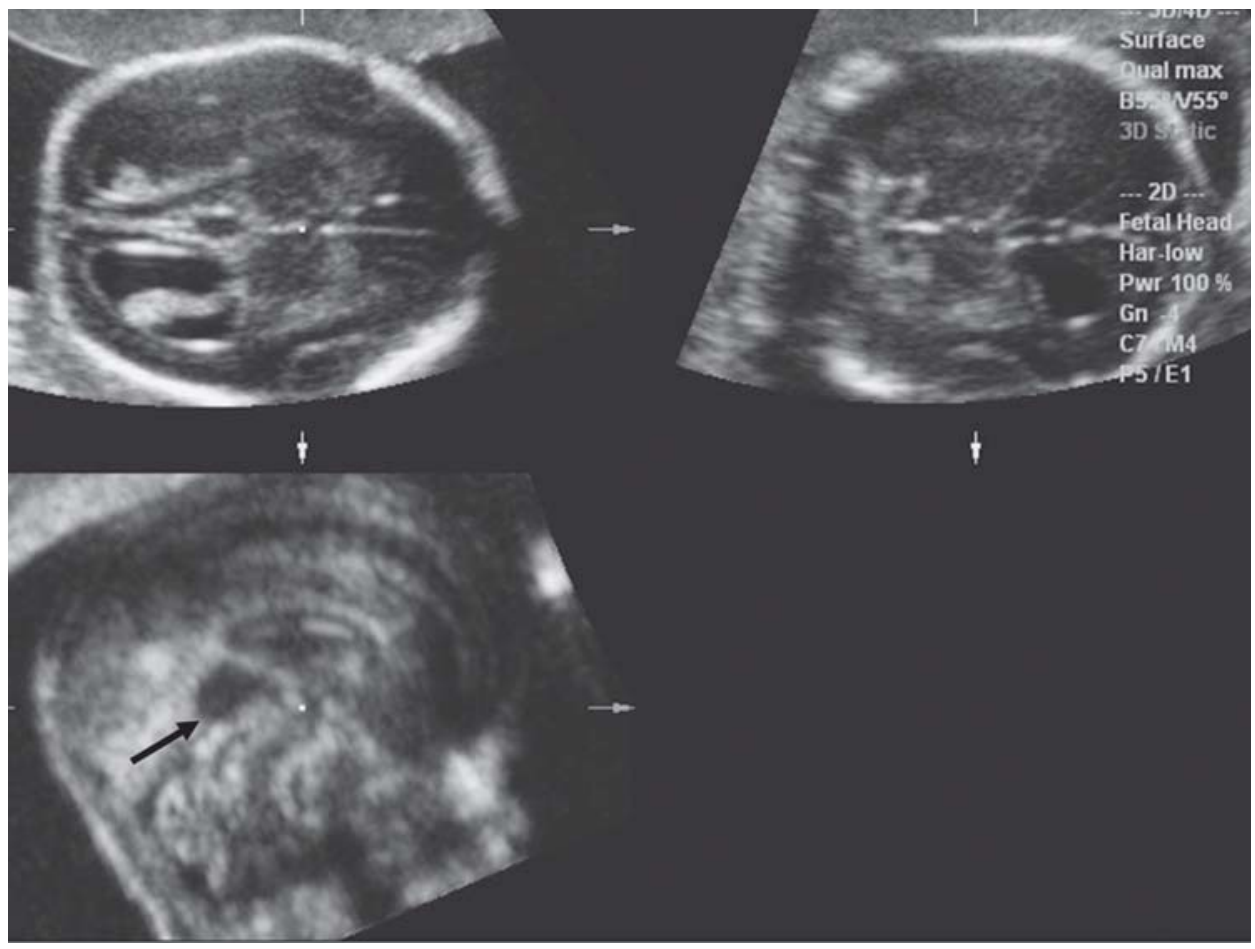

Fig. 13: Borderline ventriculomegaly associated with cavum veli interpositi cyst. The 3D multiplanar view clearly demonstrate the small cyst (arrow) located below the splenium of the corpus callosum and posterior to the cavum septi pellucidi

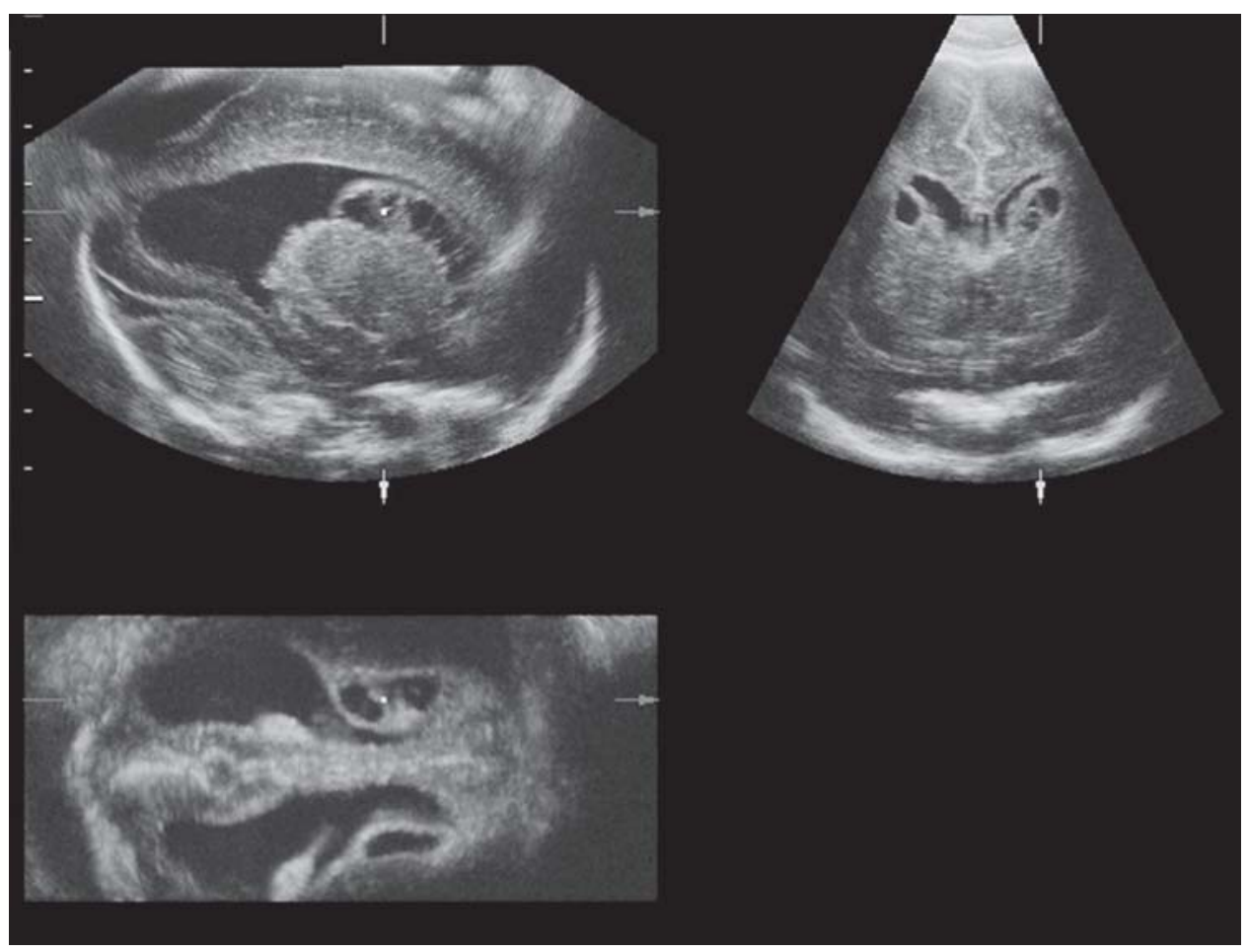

Fig. 14: Borderline ventriculomegaly associated with multiple subependymal cysts. The 3D multiplanar view clearly demonstrate the cysts located below the frontal horns of the lateral ventricles 


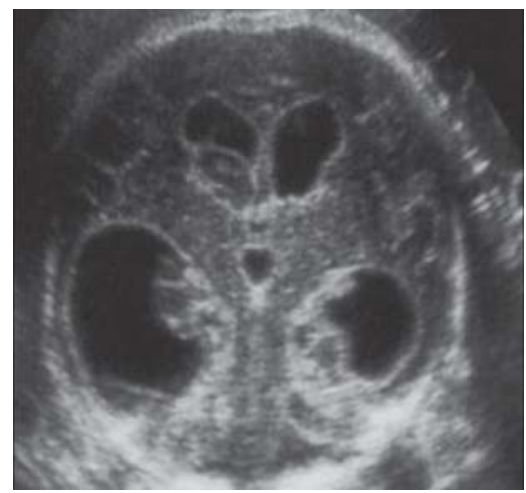

Fig. 15: Marked triventricular dilatation with signs of intraventricular hemorrhage, floating blood clots and hyperechogenic ventricular walls are visible

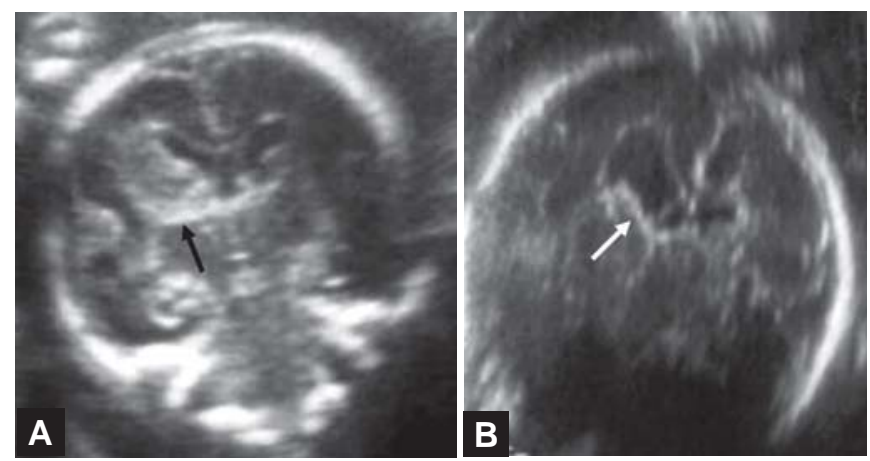

Figs 16A and B: Periventricular hemorrhage (black arrow) at 21 weeks gestation $(A)$, evolving in unilateral ventriculomegaly at 24 weeks $(B)$; the hyperechogenicity of the ventricular wall is still visible(white arrow)

The choroid plexuses should also be evaluated. In case of hypertensive ventriculomegaly they are usually squeezed by the increased cerebrospinal fluid pressure. The finding of an enlarged choroids plexus can be the sign of a papilloma (Fig. 17).

A careful examination of the brain surface should also be perfomed in order to recognize neuronal proliferation and differentiation disorders, such as lissencephaly (Fig. 18).

Finally, in cases of intrauterine infections, small periventricular echogenic areas may be seen (Fig. 19).

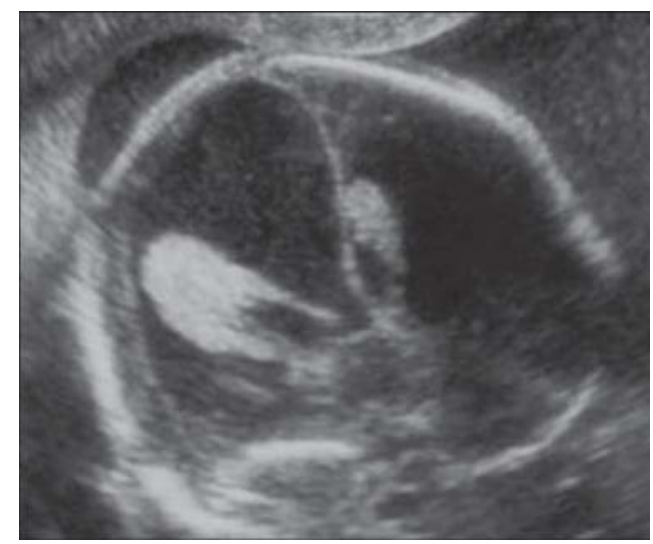

Fig. 17: Asymmetrical ventriculomegaly due to choroid plexus papilloma

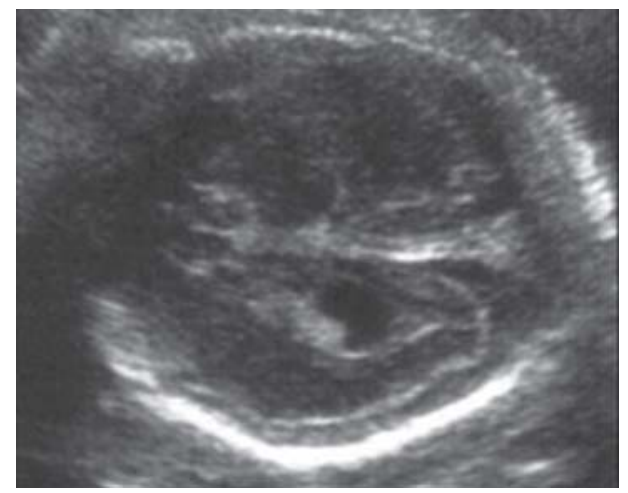

Fig. 18: Mild ventriculomegaly in a case of lissencephaly at 26 weeks of gestation. The brain surface is smooth and the arachnoidal space is large
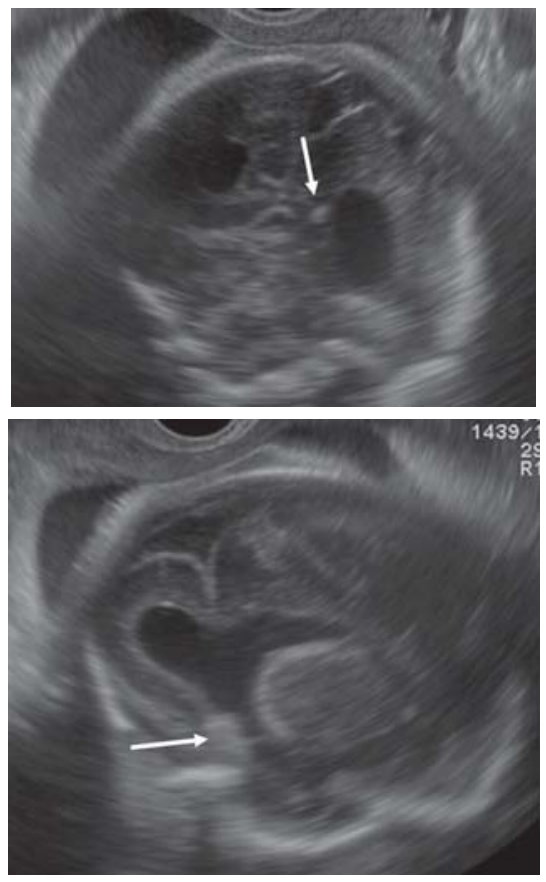

Fig 19: Mild ventriculomegaly in a case of toxoplasmosis infection. Small periventricular echogenic spots (arrows) can be visualized

\section{CONCLUSIONS}

The prenatal sonographic diagnosis of fetal ventriculomegaly is relatively easy and in cases of marked ventricular dilatation it can be achieved "at a glance" on the classical axial scans on the fetal skull. However, the recognition of the cause of the ventricular dilatation may represent a challenging problem even in the hands of experienced sonologists. The accurate and detailed evaluation of the brain anatomy is a crucial point in the differential diagnosis. The sagittal scan of the fetal brain is a powerful source of information since it allows the contemporary view of both the corpus callosum and the posterior fossa, where typical sonographic findings are present in the different causes of ventricular dilatation. The sagittal scan of 
the fetal brain is not looked for routinely; however the sonologist should be familiar with such a scan in cases of ventricul omegaly in order to obtain a more comprehensive view of the brain morphology. Furthermore he can better communicate his prenatal findings to the neonatologist, neuroradiologist and pediatric neurosurgeon, who usually eval uate the neonatal brain imaging based on the sagittal and coronal sections and can therefore better understand the prenatal sonographic features, with the final goal to offer the parents a correct counselling.

\section{REFERENCES}

1. Myrianthopoulos NC. Epidemiology of central nervous malformations. In: V inken PJ, B ruyn GW, (Eds). Handbook of clinical neurology. A msterdam: Elsevier 1977; pp 139-171.

2. M ilhorat TH. Hydrocephaly. In: V inken PJ, B ruyn GW, (Eds). Handbook of clinical neurology. A msterdam: Elsevier 1987; pp 285-300.

3. Fransen $E$, Vits $L, V$ an Camp G, Willems PJ. The clinical spectrum of mutations in $L 1$, a neuronal cell adhesion molecule. A m J M ed Genet 1996;64:73-77.

4. MCLone DG, Naidich TP. Development morphology of the subarachnoid space, brain vasculature and continuous structures, and the cause of the Chiari II malformation. A m J Neuroradiol 1992;13:463-83.

5. Barkovich AJ, Kjos BO, Normal D. Revised classification of the posterior fossa cysts and cystic-like malformations based on the results of multiplanar $M R$ imaging. Am J Neuroradiol 1990;10:977-82.

6. Y oung ID. Genetics of neurodevelopmental abnormalities. In: Levene M I, Lilford RJ, B ennet MJ, Punt F, editors. Fetal and neonatal neurology and neurosurgery. London: Churchill Livingstone 1995; pp 256-261.

7. Hadlock FP, Deter RL, Park K. Real time sonography: ventricular and vascular anatomy of the fetal brain in utero. AJR 1981;136:133-37.

8. D'A ddario V, K urjak A. U Itrasound investigation of the fetal cerebral ventricles. J Perinat M ed 1985;13:67-77.

9. M onteagudo A, Reuss M L, Timor-Tritsch IE. I maging the fetal brain in the second and third trimesters using transvaginal sonography. Obstet Gynecol 1991;77:27-32.

10. Timor-Tritsch IE, M onteagudo A, W arren W B. Transvaginal ultrasonic definition of the central nervous system in the first and early second trimester. Am J Obstet Gynecol 1991;164: 747-53.

11. J eanty $P, D$ ramix-W ilmet $M$, Del beke D. U Itrasonic eval uation of fetal ventricular growth. Neuroradiology 1981;21:127-131.

12. Hata $T, Y$ anagihara $T, M$ atsumoto $M$, Hanaoka $U$, U eta $M$, Tanaka $Y$. Three-dimensional sonographic features of fetal central nervous system anomaly. A cta Obstet Gynecol Scand 2000;79:635-39.
13. Pooh RK, Pooh K H. The assessment of fetal brain morphology and circulation by transvaginal 3D sonography and power Doppler. J Perinat M ed 2002;30:48-56.

14. Pilu G, Reece EA, Goldstein I, Hobbins J C, Bovicelli L. Sonographic evaluation of the normal developmental anatomy of the cerebral ventricles: II. The atria. Obstet Gynecol 1989;73:250-255.

15. M onteagudo A, Timor-Tritsch IE, M oomij M. Nomograms of the fetal lateral ventricles using transvaginal sonography. J Ultrasound M ed 1993;5:265-69.

16. Gupta JK, Bryce FC, Lilford RJ. M anagement of apparently isolated fetal ventriculomegaly. Obstet Gynecol Surv 1994;49:716-21.

17. Ecker J L, Shipp TD, B romley B, B enacerraf C. The sonographic diagnosis of Dandy-W alker and Dandy-W alker variant: associated findings and outcomes. Prenat Diagn 2000;20:32.

18. Goodyear PW, B annister CM, R ussel S, Rimmer S. O utcome in prenatally diagnosed fetal agenesis of the corpus callosum. F etal Diagn Ther 2001;16:139-45.

19. Davila-Gutierrez G. A genesis and dysgenesis of the corpus callosum. Sem Pediatr Neurol 2002;9:292-93.

20. Nyberg DA, M ack LA, Hirsch J, Pagon RO, Shepard TH. Fetal hydrocephalus: sonographic detection and clinical significance of associated anomalies. Radiology 1987;163:187-91.

21. Nicolaides KH, Berry S, Snijders RJ. Fetal lateral cerebral ventriculomegaly: associated malformations and chromosomal defects. Fetal Diagn Ther 1990;5:5-14.

22. Schwanitz G, Schuler H, Gembruch U, Zerres K. Chromosomal findings in fetuses with ultrasonographically diagnosed ventriculomegaly. A nn Genet 1993;36:150-53.

23. Pilu G, Falco P, Gabrielli S, Perolo A, Sandri F, Bovicelli L The clinical significance of fetal isolated cerebral borderline ventriculomegaly: report of 31 cases and review of the literature.UItrasound Obstet Gynecol. 1999; 14:320-26.

24. Gaglioti P, Danelon D, B ontempo S, M ombrò M, Cardaropoli S, Todros T. Fetal cerebral ventriculomegaly: outcome in 176 cases. Ultrasound Obstet Gynecol 2005; 25:372-77.

25. Signorelli M, Tiberti A, Valseriati D, M olin E, Cerri V, Groli C, Bianchi U A .: W idth of the lateral ventricular atrium between 10 and $12 \mathrm{~mm}$ : a simple variation of the norm? Ultrasound Obstet Gynecol. 2004; 23:14-18

26. Senat M V, B ernard J P, Delezoide A, Saugier-V eber P, Hillion $Y, R$ oume $J$. Prenatal diagnosis of hydrocephalus-stenosis of the aqueduct of Sylvius by ultrasound in the first trimester of pregnancy. Report of two cases. Prenat Diagn 2001;21:1129-32.

27. Timor-Tritsch IE, M onteagudo A, H aratz-Rubinstein N, L evine RU. Transvaginal sonographic detection of adducted thumbs, hydrocephalus, and agenesis of the corpus callosum at 22 postmenstrual weeks: the masa spectrum or $L 1$ spectrum. A case report and review of the literature. Prenat Diagn 1996;16:543-45.

28. Nicolaides K H, Campbell S, Gabbe SG, Guidetti R. U Itrasound screening for spina bifida: cranial and cerebellar signs. Lancet 1986;328:72-76. 
29. D’A ddario V, Pinto V, Del Bianco A, Di Naro E, Tartagni M, $M$ iniello G. The clivus-supraocciput angle: a useful measurement to evaluate the posterior fossa and to diagnose Chiari II malformation. U Itrasound O bstet Gynecol 2001;18:146-149.

30. Pilu G, Romero R, De Palma L. A ntenatal diagnosis and obstetrical management of Dandy-Walker syndrome. J Reprod M ed 1986;31:1017-22.

31. B ennet $G L, B$ romley $B, B$ enacerraf $B$ R. A genesis of the corpus callosum: prenatal detection usually is not possible before 22 weeks of gestation. Radiology 1996;99:447-50.
32. Pilu G, Sandri F, Perolo A, Pittalis M C, Grisolia G, Cocchi G. Sonography of fetal agenesis of the corpus callosum: a survey of 35 cases. Ultrasound O bstet Gynecol 1993;3:318-329.

33. D'A ddario V , Pinto V, Di Cagno L, Pintucci A. The midsagittal view of the fetal brain: a useful landmark in recognizing the cause of fetal cerebral ventriculomegaly. J Perinat M ed 2005;33:423-24

34. D'A ddario V , Selvaggio S, Pinto V, Resta M, Di Cagno L, Famà A: Fetal subependymal cysts with normal neonatal outcome. Fetal Diag Ther 2003;18:170-73 